\title{
Effect of optical losses irregularity on wideband fiber Raman amplification for total telecom window in silica fibers
}

\author{
G.S. Felinskyi ${ }^{1}$, M.Y. Dyriv², P.A. Korotkov ${ }^{3}$ \\ Taras Shevchenko Kyiv National University, \\ 2, Academician Glushkov prospect, 03022 Kyiv, Ukraine, \\ Phone: +38-044-526-0570, fax: +38-044-526-1073, \\ E-mail:.'felinskyi@yahoo.com, ${ }^{2}$ dyrivm@ukr.net, ${ }^{3}$ pak@mail.univ.kiev.ua
}

\begin{abstract}
Raman threshold pump power and optical amplification band calculations adjusted for attenuation dispersion in the range of total $\mathrm{S}+\mathrm{C}+\mathrm{L}$ telecom window of silica fibers (roughly from 1.46 to $1.62 \mu \mathrm{m}$ ) are presented. It has been shown that wavelength nonuniformity of signal attenuation coefficient essentially effects on amplification bandwidth and leads to the bandwidth decrease from 0.9 to $2.5 \mathrm{THz}$ depending on a pump spectral position and fiber type. Offered has been the technique of analytical approximation of attenuation coefficient as a basis for pump power threshold determination and for preceding data correction with reference to an optical amplification band. Simulation results for three widely used fibers, namely, DCF, TrueWaveRS ${ }^{\mathrm{TM}}$ and SMF have been presented. However, the technique allows estimating the optimal pump power in fiber Raman amplifiers for other fiber types as well.
\end{abstract}

Keywords: Raman amplifier, threshold pump power, single-mode fiber, optical amplification band.

Manuscript received 25.12.12; revised version received 06.02.13; accepted for publication 19.03.13; published online 25.06.13.

\section{Introduction}

Fiber Raman amplifiers (FRAs) are obligatory components of long-haul fiber-optical systems in modern telecommunications with terabit information exchange rates. In fact, due to their implicit advantages FRAs have edged legacy erbium-doped fiber amplifiers (EDFA) out of these systems [1]. Just FRAs give the unprecedented possibility of practical familiarization with the total telecommunication $\mathrm{S}+\mathrm{C}+\mathrm{L}$ frequency window (with broadening the channel information volume up to more than $20 \mathrm{THz}$ ) [2]. Nevertheless, the simplicity of FRAs realization is accompanied by a huge intricacy of nonlinear processes of energy interchange between several pump waves and hundreds of signal waves in stimulated Raman scattering (SRS), which strongly complicates the synthesis of these amplifiers [3].
The consequent solution of the FRA synthesis problem requires amplification band determination, which, by-turn, leads to direct calculating pump power threshold conditions for Raman amplification of optical signals. It should be noted that the Raman threshold introduced in classical works $[4,5]$ refers to single-pass generation that appears from Stokes noise in the absence of external optical signals. This approach is conventional, especially for analyzing Raman lasers [6, 7]. Though, it is absolutely unacceptable for FRAs, in which the consideration of external signal amplification without self-oscillation by own noises is required $[8,9]$. Threshold conditions for real Raman gain profiles (RGP) were obtained in the works $[8,9]$ in approximation of the constant attenuation coefficient $\alpha_{s}$ that requires correction in the range of operating amplification band 1.45 to $1.65 \mu \mathrm{m}$. The dispersion of optical losses in fiber and influence of this dispersion over the amplification 
band were not taken into consideration in [6-10]. Therefore, the purpose of our work is to develop pump power threshold calculation methods adjusted for signal attenuation coefficient dispersion and to analyze the threshold nature change effect on wideband Raman amplification of optical signals in the main types of silica fibers.

\section{Theoretical model}

In the process of fiber Raman amplification, as known $[11,12]$, the Raman gain coefficient $g_{R}(\omega)$ is maximum for the frequency shifted from the pump frequency approximately by $13.2 \mathrm{THz}$ (or $440 \mathrm{~cm}^{-1}$ ) when using silica fibers (see Fig. 1). As a result, the signal on the frequency that is less than the pump frequency by $13.2 \mathrm{THz}$ will be amplified as much as possible, and all the other signals with vicinal frequencies will be amplified weaker. From the modern viewpoint, the pump power threshold is defined as a pump power, at which the Raman signal gain compensates signal own losses, when the signal propagates along the fiber $[9,11]$. Thus, the fiber becomes practically transparent for a signal wave. According to this definition, the pump power threshold is a function of Raman gain coefficient and signal attenuation coefficient. The quantitative condition for the pump power threshold follows from the following system of coupled differential equations $[11,13]$ :

$$
\left\{\begin{array}{l}
\frac{d P_{s}(z)}{d z}=g_{R}(\omega) P_{p} P_{s}-\alpha_{s}(\omega) P_{s} \\
\frac{d P_{p}(z)}{d z}=-g_{R}(\omega) P_{p} P_{s} \frac{\lambda_{s}}{\lambda_{p}}-\alpha_{p}(\omega) P_{p},
\end{array}\right.
$$

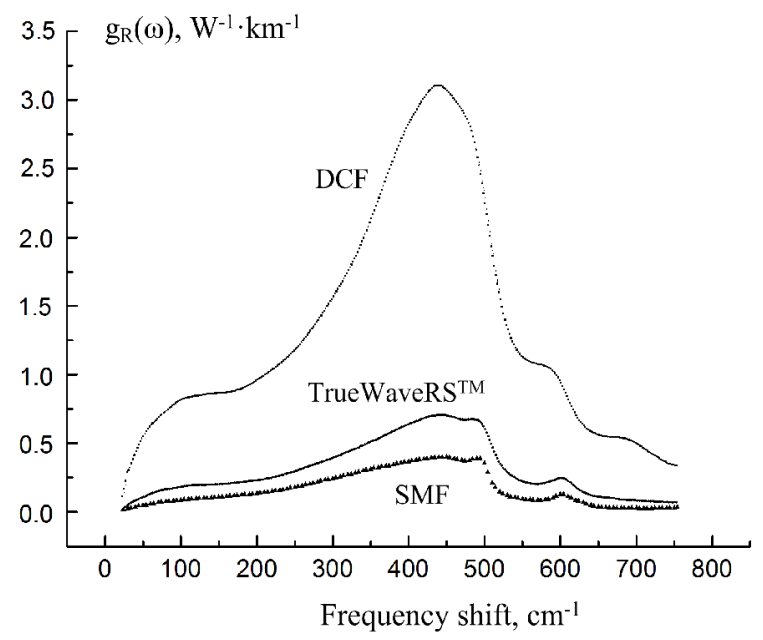

Fig. 1. Raman gain profiles in main types of silica fibers: DCF (dispersion compensating fiber), TrueWaveRS ${ }^{\mathrm{TM}}$ (nonzero dispersion shifted fiber) SMF (single mode fiber). where $P_{s}, P_{p}$ are the signal and pump powers; $\alpha_{s}, \alpha_{p}$ attenuation coefficients for signal and pump waves, respectively; $\lambda_{s}, \lambda_{p}-$ signal and pump wavelengths, respectively; $z$ is the axis along the fiber length. From the first equation of the system (1), on the assumption that the pump power $P_{p}=$ const and $\frac{d P_{s}(z)}{d z}=0$ on the certain signal frequency (wavelength), the pump power threshold will be as follows:

$P_{p}^{t h}=\frac{\alpha_{S}(\omega)}{g_{R}(\omega)}$

where $\alpha_{s}(\omega)$ is the attenuation coefficient of signal, $g_{R}(\omega)$ - the Raman gain coefficient normalized by the fiber effective area of signal-pump interaction $A_{\text {eff }}$. So, the expression (2) is a critical condition between signal wave attenuation and wave amplification in the fiber. The value inversely proportional to threshold pump power (2) is a figure of merit (FOM) of FRA [14, 15] that, in fact, characterizes the amplification quality in the fiber: the bigger is the gain and the smaller is attenuation, the higher is FOM and, correspondingly, the lower is Raman threshold. The system (1) is simplified, as it takes into account interaction between a single pump wave and a single signal wave only. Therefore, the pump power threshold is minimal only for this specific signal. For amplification of other signals within the wavelength range from 1.46 up to $1.62 \mu \mathrm{m}$, a stronger pump power is required, which follows from the equation (2) and is evident from Fig. 1. To determine the threshold pump power, analytical presentation of both $g_{R}(\omega)$ and $\alpha_{S}(\omega)$ is required. For immediate reproduction of these parameters from experimental data, we applied polynomial approximation, which consists in substitution of experimental curves with the theoretical ones where the polynomial order determines approximation errors.

\section{Results and discussion}

To calculate threshold pump power we used wavelength dependences of Raman gain profiles and signal attenuation coefficients. The calculation technique was as follows. The Raman gain coefficient $g_{R}(\omega)$ for the fibers SMF, DCF and TrueWaveRS ${ }^{\mathrm{TM}}$ is digitized from the measured data [15]. The parameter $g_{R}(\omega)$ is reproduced graphically and analytically with applying the Gauss spectral decomposition method with minimal approximation errors [17]. The profiles curves are shown in Fig. 1. The value of the frequency shift in $\mathrm{cm}^{-1}$ is plotted on X-axis. The signal wavelength dependence of the coefficient $\alpha_{S}(\lambda)$ is also presented in a digital form from the data [15] for the same fiber types. We realized polynomial approximation of the investigated curves for the signal attenuation coefficient $\alpha_{s}=\alpha_{s}\left(\lambda_{s}\right)$ : 

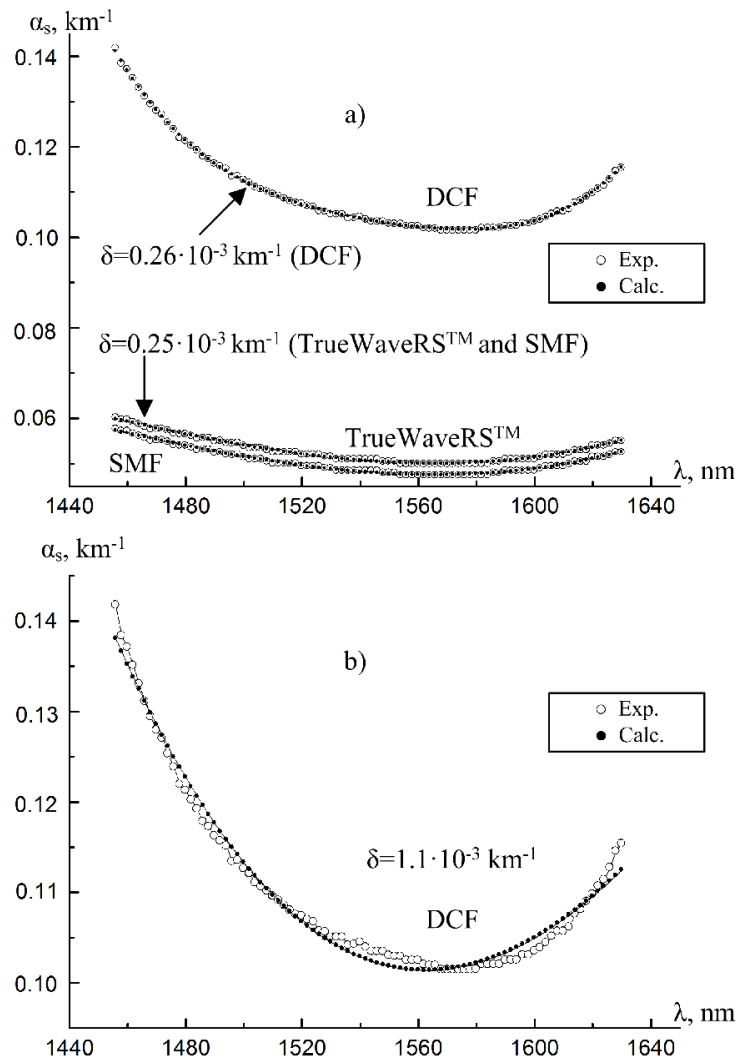

Fig. 2. Signal attenuation coefficient $\alpha_{s}$ as a function of the signal wavelength for three types of fibers (a). 3-rd order polynomial approximation of $\alpha_{S}(\lambda)$ for DCF in the case of default of criterion (4) is shown in the inset on the right (b). Initial data are marked by light circles, calculated - dark circles.

$$
\alpha_{s}\left(\lambda_{s}\right)=\alpha_{s}^{\min }\left[1+\sum_{k=1}^{n} \alpha_{k}\left(\frac{\lambda_{s}-\lambda_{s, \alpha_{s}^{\min }}}{\lambda_{s}}\right)^{k}\right],
$$

where $\alpha_{s}^{\min }$ and $\lambda_{s, \alpha_{s}^{\min }}$ are minimal values of the attenuation and respective signal wavelength, $\alpha_{k}-$ polynomial coefficients, $\lambda_{s}$ is the signal wavelength. So, as the value of the attenuation coefficient $\alpha_{S}(\lambda)$ in SMF is practically the same as in the fiber TrueWaveRS ${ }^{\mathrm{TM}}$, then the least order of polynomial that satisfactorily approximates digitized data $\alpha_{s}=\alpha_{s}\left(\lambda_{s}\right)$ in the case of SMF and TrueWaveRS ${ }^{\mathrm{TM}}$ is the 3 -rd one, but in the case of DCF (dispersion compensating fiber) it is the 4-th one. The criterion of usability of polynomial approximant with either that or another order is the empirical condition introduced by us previously [17]. It has the following expression:

$\eta=\frac{\delta}{Q_{\text {mean }}} \leq 5 \cdot 10^{-3}$,

where $\delta$ is the standard approximation error, $Q_{\text {mean }}-$ average value of approximating quantity. In particular, the standard error of $\alpha_{S}(\lambda)$ approximation by the 3-rd order polynomial is $\delta>1.1 \cdot 10^{-3} \mathrm{~km}^{-1}$ in DCF, which dissatisfies the condition (4) (look at Fig. 2b). Therefore, for that fiber we made $\alpha_{s}(\lambda)$ approximation by the 4-th order polynomial with $\delta=2.6 \cdot 10^{-4} \mathrm{~km}^{-1}$, which corresponds to $\eta=2.4 \cdot 10^{-3} \approx 0.2 \%$. The parameters of polynomial approximation of $\alpha_{s}=\alpha_{s}\left(\lambda_{s}\right)$ (Eq. (3)) are given in Table 1 for these three investigated fibers. The calculated dependences $\alpha_{s}=\alpha_{S}\left(\lambda_{s}\right)$ for SMF, DCF and TrueWaveRS ${ }^{\mathrm{TM}}$ are plotted in Fig. 2a.

We have obtained wavelength dependent threshold pump power values by substituting the calculated analytical expressions for the quantities $g_{R}\left(\lambda_{s}\right)$ and $\alpha_{s}\left(\lambda_{s}\right)$ into Eq. (2). The wavelength dependences $P_{p}^{t h}=P_{p}^{t h}\left(\lambda_{s}\right)$ for these fibers are plotted in Fig. 3 . When the pump power exceeds the threshold one, signal amplification is observed in the wide range of wavelengths (the amplification domain is located above the level of the correcting line of threshold pump power marked by dark triangles in Fig. 3): approximately from 1.46 to $1.62 \mu \mathrm{m}$ (or $20.3 \mathrm{THz}$ ) depending on a fiber type and pump power value. We made some corrections to the pump power threshold taking signal attenuation dispersion into consideration. The pump power threshold dependences on wavelength when $\alpha_{s}=\alpha_{S}\left(\lambda_{s}\right)$ and also when $\alpha_{s}=$ const $=0.45 \mathrm{~dB} / \mathrm{km}\left(\approx 0.1 \mathrm{~km}^{-1}\right)$ in DCF are shown in Fig. 3a. In this case, the minimal pump power threshold is close to $\mathbf{3 3} \mathbf{~ m W}$ at the wavelength $1552 \mathrm{~nm}$. For the fibers TrueWaveRS ${ }^{\mathrm{TM}}$ and SMF (Figs $3 b$ and 3c, respectively) at this wavelength, there are the following least threshold powers: $P_{p_{\min }(T W R S)}^{\text {th }} \approx 71 \mathrm{~mW} \quad\left(\alpha_{s}=0.21 \mathrm{~dB} / \mathrm{km}\right) \quad$ and $P_{p_{\min }(S M F)}^{\text {th }} \approx 119 \mathbf{~ m W} \quad\left(\alpha_{s}=0.21 \mathrm{~dB} / \mathrm{km}\right) . \quad \mathrm{A}$ significant discrepancy between the calculated curves is detected at the wavelengths near edges of the investigated wavelength range. For example, at the wavelength $1462 \mathrm{~nm}$ the difference between the curves $\alpha_{s}=\alpha_{S}\left(\lambda_{s}\right)$ and $\alpha_{s}=$ const is higher than $200 \mathbf{~ m W}$ in SMF, - higher than $100 \mathbf{~} \mathbf{~ W}$ in the dispersion shifted fiber TrueWaveRS ${ }^{\mathrm{TM}}$, and higher than $\mathbf{6 0} \mathbf{~ m W}$ in DCF. At the wavelength $1576 \mathrm{~nm}$, by-turn, the received curves differ from each other not more than $\mathbf{5} \mathbf{~ m W}$ for SMF and TrueWaveRS ${ }^{\mathrm{TM}}$, and for DCF, they nearly coincide. It is explained by the nature of attenuation losses for the signal wave: the value of the attenuation coefficient $\alpha_{s}$ in the given fibers is larger near the edges than in the centre of the investigated range 1.46 to $1.62 \mu \mathrm{m}$ and, accordingly, the threshold would be larger in comparison with the case when $\alpha_{s}=$ const. As a result, the pump power threshold increase leads to amplification bandwidth reduction at the constant value of pump 
Table 1. Coefficients of polynomial approximation of experimental curves for the signal attenuation coefficient $\alpha_{\mathrm{s}}=$ $\alpha_{s}\left(\lambda_{s}\right)$ in typical silica fibers.

\begin{tabular}{|c|c|c|c|c|c|c|}
\hline Fiber types & $\alpha_{s}^{\min }, \mathrm{km}^{-1}$ & $\lambda_{s, \alpha_{s}^{\min }}, \mathrm{nm}$ & $\alpha_{1}$ & $\alpha_{2}$ & $\alpha_{3}$ & $\alpha_{4}$ \\
\hline DCF & 0.102 & 1574 & 0.098 & 65.36 & 1062.431 & 12077.762 \\
\hline TrueWaveRS & 0.050 & 1570 & 0.309 & 55.726 & 253.595 & - \\
\hline SMF & 0.047 & 1570 & 0.327 & 58.702 & 266.812 & - \\
\hline
\end{tabular}

Table 2. Effect of threshold pump powers correction on the signal amplification band in operation wavelength range of the total $\mathbf{S}+\mathrm{C}+\mathrm{L}$ telecom window in the fiber $\mathrm{DCF}$.

\begin{tabular}{|c|c|c|c|c|}
\hline$P_{p}, \mathrm{~mW}$ & $\lambda_{p}, \mathrm{~nm}$ & $B W_{b e f}, \mathrm{THz}$ & $B W_{a f t}, \mathrm{THz}$ & $\Delta_{B W}, \mathrm{THz}$ \\
\hline \multirow{5}{*}{60} & 1407 & 6.17 & 5.21 & 0.96 \\
\hline & 1429 & 6.1 & 5.31 & 0.79 \\
\hline & 1451 & 5.9 & 5.52 & 0.38 \\
\hline & 1473 & 5.6 & 5.47 & 0.13 \\
\hline & 1495 & 5.42 & 5.3 & 0.12 \\
\hline \multirow{3}{*}{100} & 1429 & 12.4 & 10.8 & 1.6 \\
\hline & 1451 & 11.9 & 10.9 & 1 \\
\hline & 1473 & 11.6 & 10.8 & 0.8 \\
\hline \multirow{2}{*}{125} & 1451 & 14.8 & 12.3 & 2.5 \\
\hline & 1473 & 14.4 & 12.4 & 2 \\
\hline \multirow{2}{*}{150} & 1451 & 16.4 & 15.1 & 1.3 \\
\hline & 1473 & 15.8 & 14.9 & 0.9 \\
\hline 200 & 1451 & 18.9 & 18 & 0.9 \\
\hline
\end{tabular}

power. Thus, we have received the next corrections to amplification band for the presented single-mode silica fibers: for SMF at the pump power $500 \mathrm{~mW}$, the amplification bandwidth reduces by $0.9 \mathrm{THz}$; for TrueWaveRS ${ }^{\mathrm{TM}}$ - by $1.2 \mathrm{THz}$ at $250 \mathrm{~mW}$; and for DCF by $2.5 \mathrm{THz}$ at $125 \mathrm{~mW}$. These different pump powers, for which we have marked the difference between the curves (see Fig. 3), are illustrated by the Raman gain coefficient value in each of fibers. The dispersion compensating fiber is the most optimal fiber among all the investigated ones from the figure of merit viewpoint. We exposed the results of the effect of the corrected curves of the pump power threshold in view of $\alpha_{s}=\alpha_{s}\left(\lambda_{s}\right)$ on optical amplification band in DCF in detail in Table 2: $P_{p}$ is the pump power, $\lambda_{p}$ - investigated pump wavelengths, $B W_{\text {bef }}$ - amplification bandwidth before correction of $P_{p}^{\text {th }}, B W_{\text {aft }}-$ amplification bandwidth after correction of $\quad P_{p}^{t h}, \quad \Delta_{B W}=B W_{b e f}-B W_{a f t} \quad-$ amplification bandwidth reduction.

The maximal amplification bandwidth reduction is obtained when pump power is derived in DCF at lower wavelengths from the whole investigated range 1400$1500 \mathrm{~nm}$, as shown in Table 2. In this fiber at the pump power $125 \mathrm{~mW}$, the amplification bandwidth reduces from 14.8 to $12.3 \mathrm{THz}$, or becomes narrower almost by $17 \%$. Thus, the realized pump power threshold calculation is substantial for finding the amplification band. Having analyzed the obtained results, we found that increasing pump power above threshold $P_{p}^{t h}$ leads not only to signal power rise, but to the rise of amplified signal numbers in the range from 1.46 to $1.62 \mu \mathrm{m}$. It becomes evidently that the pump power threshold must be the same for all the signals within the investigated range for their uniform amplification and this, in its turn, requires application of multimode pumps. 

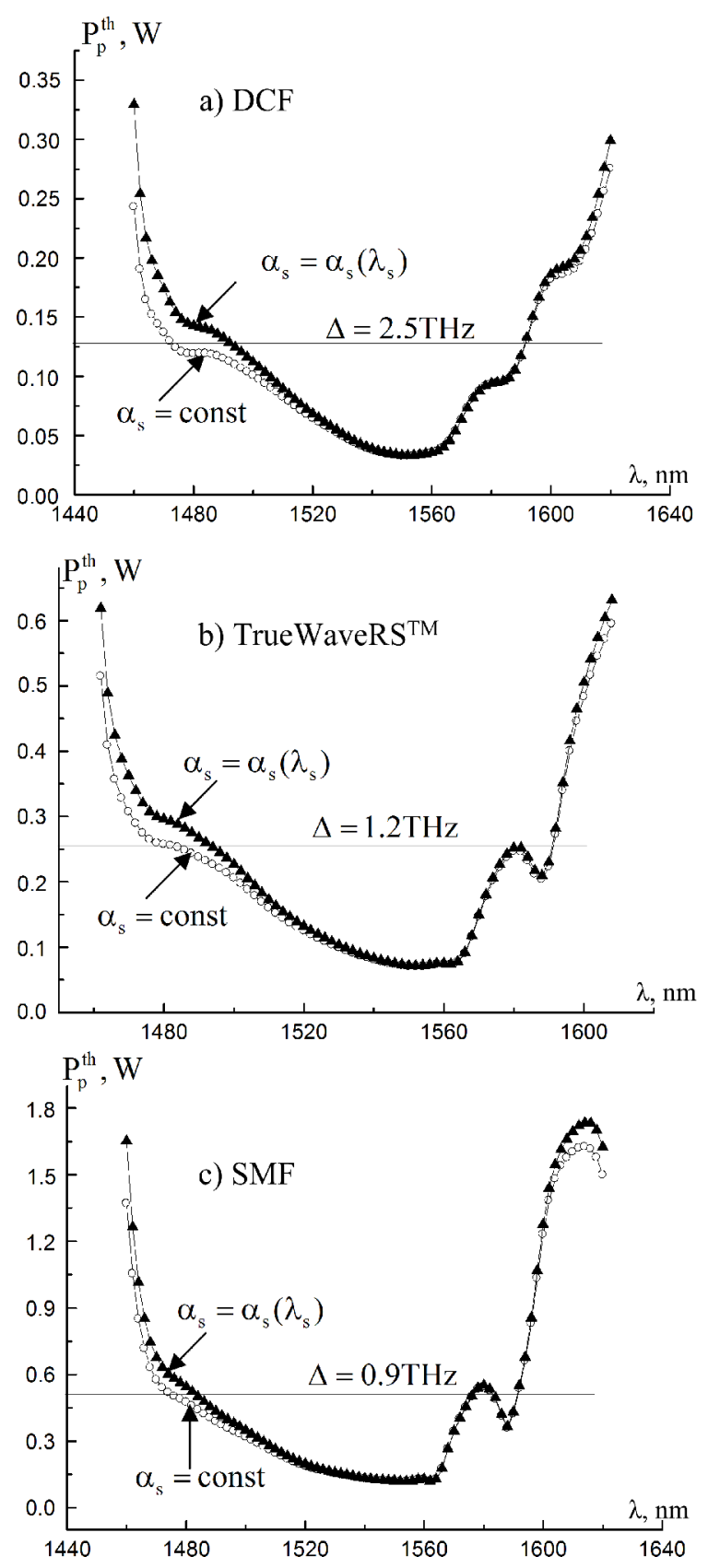

Fig. 3. Updating the pump power threshold at the edges of the range $1.46 \ldots 1.62 \mu \mathrm{m}$ in the fibers DCF (a), TrueWaveRS ${ }^{\mathrm{TM}}$ (b) and SMF (c).

\section{Conclusion}

Thus, the wavelength irregularity of the signal attenuation effect on threshold pump powers in optical single-mode fibers has been investigated in our work. This effect results in Raman amplification bandwidth contraction in a wide range of wavelengths, which covers telecom $\mathrm{S}+\mathrm{C}+\mathrm{L}$ windows:

1. The calculating technique of threshold pump power determination in fiber-optics Raman amplifiers has been proposed. The technique takes into account signal attenuation dispersion in optical fibers and is based on analytical expressions as for Raman gain coefficient $g_{R}(\lambda)$, as for attenuation coefficient $\alpha_{S}(\lambda)$ of useful signal. We have derived the polynomial equation for approximation of experimental data of attenuation coefficient dependence on wavelength. It was determined that approximation accuracy $0.5 \%$ is provided by polynomials of the order $n \leq 4$ for the three widely used silica fibers - DCF, TrueWaveRS ${ }^{\mathrm{TM}}$ and SMF.

2. Adjusted threshold pump power dependences of signal wavelength have been obtained. A significant discrepancy of threshold characteristics from approaching $\alpha_{S}=$ const is clearly observed at wavelengths near the edges of the investigated wavelength range and has the maximum value more than $200 \mathrm{~mW}$ in SMF, $>100 \mathrm{~mW}$ in TrueWaveRS ${ }^{\mathrm{TM}}$ and $>60 \mathrm{~mW}$ in DCF at the signal wavelength $1462 \mathrm{~nm}$, which corresponds to the pump wavelength $1374 \mathrm{~nm}$.

3. Wideband Raman threshold correction with account of attenuation dispersion in optical fibers is necessary, since any fixed pump power exceeding threshold power uniquely defines frequency position of full fiber transparency points that, in fact, are the limits of the amplification band. Thus, the respective corrections to amplification band are as follows: for SMF $-0.9 \mathrm{THz}$, for TrueWaveRS $\mathrm{S}^{\mathrm{TM}}$ - approximately $1.2 \mathrm{THz}$ and for DCF $-2.5 \mathrm{THz}$ (i.e. $\approx 17 \%$ of the total bandwidth).

Hereby, our analysis allows to estimate the instant (static) Raman amplification band under arbitrary pump distribution in fixed fiber cut and is helpful for creating a generalized dynamic model of FRAs and solving synthesis tasks for such devices.

\section{References}

1. J. Bromage, Raman amplification for fiber communications systems // J. Light. Techn., 20(1), p. 79-93 (2004).

2. C. Rivero, High gain/broadband oxide glasses for next generation Raman amplifiers: Diss. for the degree of PhD, Univ. of Central Florida, 2005.

3. H. Kidorf, K. Rottwitt, M. Nissov, M. Ma, and E.Rabarijaona // IEEE Phot. Techn. Lett., 11(5), p. 530-532 (1999).

4. C. Lin, R.H. Stolen, W.G. French and T.G. Malone, A cw tunable near-infrared (1.085-1.175- $\mu \mathrm{m})$ Raman oscillator // Opt. Lett., 1(3), p. 96-97 (1977).

5. R.H. Stolen, C. Lin, J. Shan and R.F. Leheny, A fiber Raman ring laser // IEEE J. Quant. Electron., 14(11), p. 860-862 (1978).

6. A. Bertoni, G.C. Reali, 1.24- $\mu$ m cascaded Raman laser for 1.31- $\mu \mathrm{m}$ Raman fiber amplifiers // Appl. Phys. B, 67, p. 5-10 (1998). 
7. S.D. Jackson, P.H. Muir, Theory and numerical simulation of $n$-th order cascaded Raman fiber lasers // J. Opt. Soc. Am. B, 18(9), p. 1297-1306 (2001).

8. G.S. Felinskyi, P.A. Korotkov, Raman threshold and optical gain bandwidth in silica fibers // Semiconductor Physics, Quantum Electronics \& Optoelectronics, 11(4), p. 360-363 (2008).

9. G.S. Felinskyi, P.A. Korotkov, Lasing threshold for stimulated Raman generation of monochrome optical wave in single mode fibers // Proc. 7th Intern. Conf. on Laser and Fiber-Optical Networks Modeling, June 29 - July 1, 2006, Kharkiv, p. 110 112.

10. A.S. Samra, H.A.M. Harb, Wide band flat gain Raman amplifier for DWDM communication systems // Proc. IFIP Intern. Conf. on Wireless and Optical Comm. Networks, April 28-30, Cairo, p. 15 (2009).

11. P.A. Korotkov, G.S. Felinskyi, Forced-Ramanscattering-based amplification of light in one-mode quartz fibers // Rev. Ukr. J. Phys. 5(2), p. 103-169 (2009).
12. G.P. Agrawal, Nonlinear Fiber Optics, Second ed. Academic Press, San Diego, CA, 1995.

13. V. I. Grygoruk, P.A. Korotkov, G.S. Felinskyi, Nonlinear and Laser Processes in Optical Fibers. VPC "Kyivskyi universytet", Kyiv, 2008 (in Ukrainian).

14. M. Islam (ed.), Raman Amplifiers for Telecommunications 2: Sub-systems and Systems. Springer-Verlag, New York, 2004.

15. C. Headley, G.P. Agrawal, Raman Amplification in Fiber Optical Communication Systems. Elsevier Academic Press, San Diego, CA, 2005.

16. J. Bromage, K. Rottwitt and M.E. Lines, A method to predict the Raman gain spectra of germanosilicate fibers with arbitrary index profiles // IEEE Photonics Techn. Lett., 14(1), p. 24-26 (2002).

17. M. Dyriv, P. Korotkov, G. Felinskyi, Raman gain profile simulation in single-mode fibers using spectral decomposition // Bull. of Taras Shevchenko Nat. Univ. of Kyiv, Radiophysics and Electronics, 18, p. 15-18 (2012), in Ukrainian. 